\title{
Sobre o gênero Lophyraspis Stål e descrição de novas espécies (Hemiptera, Aetalionidae, Biturritiinae) ${ }^{1}$
}

\author{
Albino M. Sakakibara ${ }^{2,4} \&$ Antonio J. Creão-Duarte ${ }^{3}$
}

${ }^{1}$ Contribuição $n^{\circ} 1439$ do Departamento de Zoologia, Universidade Federal do Paraná.

${ }^{2}$ Departamento de Zoologia, Universidade Federal do Paraná. Caixa Postal 19020, 81531-980 Curitiba-PR, Brasil. Endereço eletrônico: sakaki@bsi.com.br

${ }^{3}$ Departamento de Sistemática e Ecologia, Universidade Federal da Paraíba. 83800-990 João Pessoa-PB, Brasil. Endereço eletrônico: creao@dse.ufpb.br

${ }^{4}$ Bolsista do CNPq.

\begin{abstract}
Aвstract. On the genus Lophyraspis Stål and description of new species (Hemiptera, Aetalionidae, Biturritiinae). The genus Lophyraspis and the following species are treated, along with some nomenclatural changes: Lophyraspis Stål, 1869 = Gerridius Fowler, 1896 syn. rest.; Lophyraspis muscaria (Fabricius, 1803) = Gerridius scutellatus Fowler, 1896 syn. nov. = Gerridius abbreviatus Baker, 1907 syn. nov.; Lophyraspis pygmaea (Fabricius, 1803); Lophyraspis scutellata $($ Fabricius, 1803 $)=$ L. cristata Stål, 1869 syn. nov. = L. armata Haviland, 1925 syn. nov.; Lophyraspis spinosa (Funkhouser, 1930) comb. nov. (formerly in Mina Walker, 1858); Lophyraspis fenestrata sp. nov. (Brazil, Amazonas); Lophyraspis diminuta sp. nov. (Brazil, Mato Grosso).
\end{abstract}

Keywords. Aetalionidae; Biturritiinae; Hemiptera; Lophyraspis; taxonomy.

Resumo. O gênero Lophyraspis e as seguintes espécies, juntamente com algumas mudanças nomenclaturais, são tratados: Lophyraspis Stål, 1869 = Gerridius Fowler, 1896 syn. rest.; Lophyraspis muscaria (Fabricius, 1803) = Gerridius scutellatus Fowler, 1896 syn. nov. = Gerridius abbreviatus Baker, 1907 syn. nov.; Lophyraspis pygmaea (Fabricius, 1803); Lophyraspis scutellata (Fabricius, 1803) $=$ L. cristata Stål, 1869 syn. nov. $=$ L. armata Haviland, 1925 syn. nov.; Lophyraspis spinosa (Funkhouser, 1930) comb. nov. (anteriormente em Mina Walker, 1858); Lophyraspis fenestrata sp. nov. (Brasil, Amazonas); Lophyraspis diminuta sp. nov. (Brasil, Mato Grosso).

Palavras-chave. Aetalionidae; Biturritiinae; Hemiptera; Lophyraspis; taxonomia.

STÅL (1869), ao redescrever as espécies de FABRICIUS (1803), criou o gênero Lophyraspis para acomodar Cicada vittata Olivier, 1790, Tettigonia pygmaea Fabricius, 1803 e T. scutellata Fabricius, 1803.

Fowler (1896) descreveu Gerridius tendo como espécietipo, G. scutellatus Fowler, 1896.

BAKER (1907) descreveu Gerridius abbreviatus, com base em um exemplar macho coletado em Nicarágua. Comentou que era semelhante à G. scutellatus Fowler, apenas um pouco menor quanto ao comprimento das asas anteriores.

HAVILAND (1925) considerou G. scutellatus Fowler como pertencente a Lophyraspis Stål. Para desfazer a homonímia resultante com L. scutellata (Fabricius), renomeou-a de Lophyraspis fowleri.

Deitz \& Dietrich (1993) designaram o lectótipo de Tettigonia muscaria Fabricius, 1803, e forneceram desenhos detalhados do espécime.

Abreviaturas utilizadas: DZUP - Departamento de Zoologia, Universidade Federal do Paraná, Curitiba-PR, Brasil; USNM - National Museum of Natural History, Smithsonian Institution, Washington-DC, USA.

Em "Material examinado" são registradas apenas as localidades.

\section{Lophyraspis Stål, 1869}

Lophyraspis Stål, 1869: 55 (espécie-tipo: Cicada vittata Olivier, 1790, por designação subseqüente = Tettigonia muscaria Fabricius, 1803); Funkhouser, 1927: 432; Metcalf \& Wade, 1965: 1536; Deitz \& Dietrich, 1993: 290; McKamey, 1998: 17.

Gerridius Fowler, 1896: 165 (espécie-tipo: G. scutellatus Fowler, 1896, por monotipia $=$ Lophyraspis fowleri Haviland, 1925); Funkhouser, 1927: 432 (= Lophyraspis Stål); Metcalf \& Wade, 1965:

1539; Deitz \& Dietrich, 1993: 290; McKamey, 1998: 17. Syn. rest.

Diagnose. Espécies com indivíduos relativamente pequenos (5 a 3,5 mm). Cabeça triangular; vértice com o bordo superior elevado acima dos olhos e afastado do tórax, de aspecto laminar, contorno distintamente bilobado, deprimido entre os ocelos. Olhos semi-globulares, pouco salientes. Ocelos inconspícuos, mais próximos dos olhos que entre si. Lóbulos suprantenais obsoletos. Pronoto curto, com a margem posterior arredondada, carena média ausente ou bem marcada e laminar, ou elevada em forma de crista. Escutelo bem desenvolvido, a porção apical elevada em crista, algumas vezes sobrepassando a altura do pronoto, com o bordo posterior cortante, translúcido, incolor, ou formando uma faixa pré-apical. Tégminas vítreas, com cinco células apicais e uma discoidal 
(Stål considerou como tendo duas células discoidais, contando a célula R como uma delas). Asas posteriores com quatro células apicais. Pernas anteriores e médias simples, as posteriores com tíbia delgada e curvada. Abdome, em geral, com os primeiros tergos esbranquiçados lateralmente.

STÅL (1869) considerou dois grupos de espécies dentro de Lophyraspis: um com o pronoto destituído de carena média e outro provido de carena média. No primeiro grupo incluiu Lophyraspis vittata (Olivier, 1790) e L. pygmaea (Fabricius, 1803) e, no segundo, L. scutellata (Fabricius, 1803).

Haviland (1925: 261) transferiu Gerridius scutellatus Fowler, 1896 para o gênero Lophyraspis Stål; devido à ocorrência de homonímia secundária com Tettigonia scutellata Fabricius, 1803, chamou-a de Lophyraspis fowleri, fazendo o seguinte comentário: "After a careful comparison of the characters determining the genera Lophyraspis Stål and Gerridius Fowler, I have come to the conclusion that the distinctions between them are not well defined, and that therefore Fowler's genus, erected in 1909 [sic], cannot stand. Mr. Funkhouser writes to me that he is also of this opinion.". Ao colocar Gerridius scutellatus Fowler, 1896 (espécie-tipo) dentro de Lophyraspis Stål, 1869, informalmente, sinonimizou Gerridius com Lophyraspis.

FunKHOUSER (1927) manteve a sua opinião, considerando Lophyraspis Stål 1869 um nome válido e tendo como seu sinônimo júnior, Gerridius Fowler, 1896.

Metcalf \& Wade (1965) e McKamey (1997; 1998) desconsideraram a sinonímia e, novamente, trataram os dois gêneros como táxons distintos.

Comparando os desenhos do lectótipo (macho) de $T$. muscaria fornecidos por DeITz \& Dietrich (1993: 292, figs. 911) com os de Gerridius scutellatus Fowler (também macho, Fowlen, 1896: P1. 10, figs. 11,a-b), verificamos que são idênticos. Conseqüentemente, Gerridius Fowler e Lophyraspis Stål são, realmente, sinônimos. No presente trabalho restauramos a sinonímia (syn. restauratum), em princípio conjeturada por HaVILAND (1925) e, depois, formalizada por FUNKHOUSER (1927).

Espécies incluídas:

A. Pronoto destituído de carena média.

Lophyraspis muscaria (Fabricius, 1803)

(Figs. 1, 2)

Cicada vittata Olivier, 1790: 762 (praeoc.).

Tettigonia muscaria Fabricius, 1803: 44 (localidade-tipo: América do Sul); Deitz \& Dietrich, 1993: 293 (desig. lectótipo)

Lophyraspis muscaria; Metcalf \& Wade, 1965: 1537; McKamey, 1998: 17 (cat.)

Gerridius scutellatus Fowler, 1896: 166 (localidade-tipo: Panamá) (praeoc.); Metcalf \& Wade, 1965: 1540 (= Lophyraspis fowleri Haviland, 1925). Syn. nov.

Gerridius abbreviatus Baker, 1907: 114 (localidade-tipo: Nicarágua); Metcalf \& Wade, 1965: 1539; McKamey, 1998: 17 (cat.). Syn. nov.

Lophyraspis fowleri Haviland, 1925: 261 (n. nov. pro G. scutellatus Fowler).
Lophyraspis abbreviatus; Funkhouser, 1927: 432.

Lophyraspis vittata; Funkhouser, 1927:432.

Gerridius fowleri; McKamey, 1997: 5; McKamey, 1998: 17 (cat.).

Diagnose. Escutelo elevado acima do nível do pronoto em uma crista vertical, mais ou menos triangular, mais alta que larga na base, arredondada no ápice; margem posterior da crista formando uma aresta laminar, cortante, translúcida e incolor. Tégminas vítreas, com áreas escuras na base, no disco e perto do ápice, e uma estreita faixa margeando a parte distal, incluindo o ápice do clavo.

Material examinado. PANAMÁ. BRASIL. Pará; Mato Grosso: Sinop; Chapada dos Guimarães. Distrito Federal: Planaltina. São Paulo: Ribeirão Preto (DZUP).

STÅL (1869) considerou Tettigonia muscaria Fabricius, 1803 como sinônimo júnior de Cicada vittata Olivier, 1790. Descobriu-se, mais tarde, que Cicada vittata Olivier estava pré-ocupado por $C$. vittata Linnaeus, 1758. Então, o nome da espécie passou a ser Lophyraspis muscaria (Fabricius, 1803), seu sinônimo,

A julgar pela descrição fornecida por BAKER (1907) para $G$. abbreviatus, na qual comenta a sua semelhança com $G$. scutellatus Fowler, e levando em conta a sua distribuição simpátrica, colocâmo-la na sinonímia.

\section{Lophyraspis pygmaea (Fabricius, 1803)} (Fig. 3)

Tettigonia pygmaea Fabricius, 1803: 44 (localidade-tipo: América do Sul); McKamey, 1998: 17 (cat.).

Lophyraspis parvimusca Goding, 1927: 188; Metcalf \& Wade, 1965: 1538 (cat.) (sin.)

Lophyraspis pygmaea; Funkhouser, 1927: 432; Metcalf \& Wade, 1965: 1537; McKamey, 1998: 17 (cat.).

Diagnose. Escutelo elevado pouco acima do nível do pronoto, mais largo na base que alto, obliquamente truncado, com o corte ascendente para trás, a margem posterior laminar, largamente recortada, translúcida e incolor. Tégminas vítreas, com uma estreita faixa escura contornando a margem distal, incluindo o ápice do clavo.

Material examinado. BRASIL. Pará. (DZUP).

STÅl (1869), quando redescreveu L. vittata (Olivier) e colocou T. muscaria Fabricius como seu sinônimo, citou também a fig. 82 do trabalho de STOLl (1788). Esta figura, que mostra o inseto em vista dorsal, não permite detectar se o escutelo forma ou não uma crista. A descrição de STOLL (loc. cit.) não menciona este caráter (que é bastante chamativo).

Goding (1927) chamou de Lophyraspis parvimusca Stoll a espécie descrita e figurada por STOLL (1788: Pl. 15 fig. 82F) mas que o autor a denominara de "Cicada petite mouche", e colocou como seu sinônimo T. pygmaea Fabricius, 1803. Acontece que Goding (loc. cit.) ao dar o nome correto à espécie de Stoll, tornou-se o autor da mesma e, em conseqüencia, L. pygmaea Fabricius passou a ser o nome válido (Metcalf \& WADE 1965). 


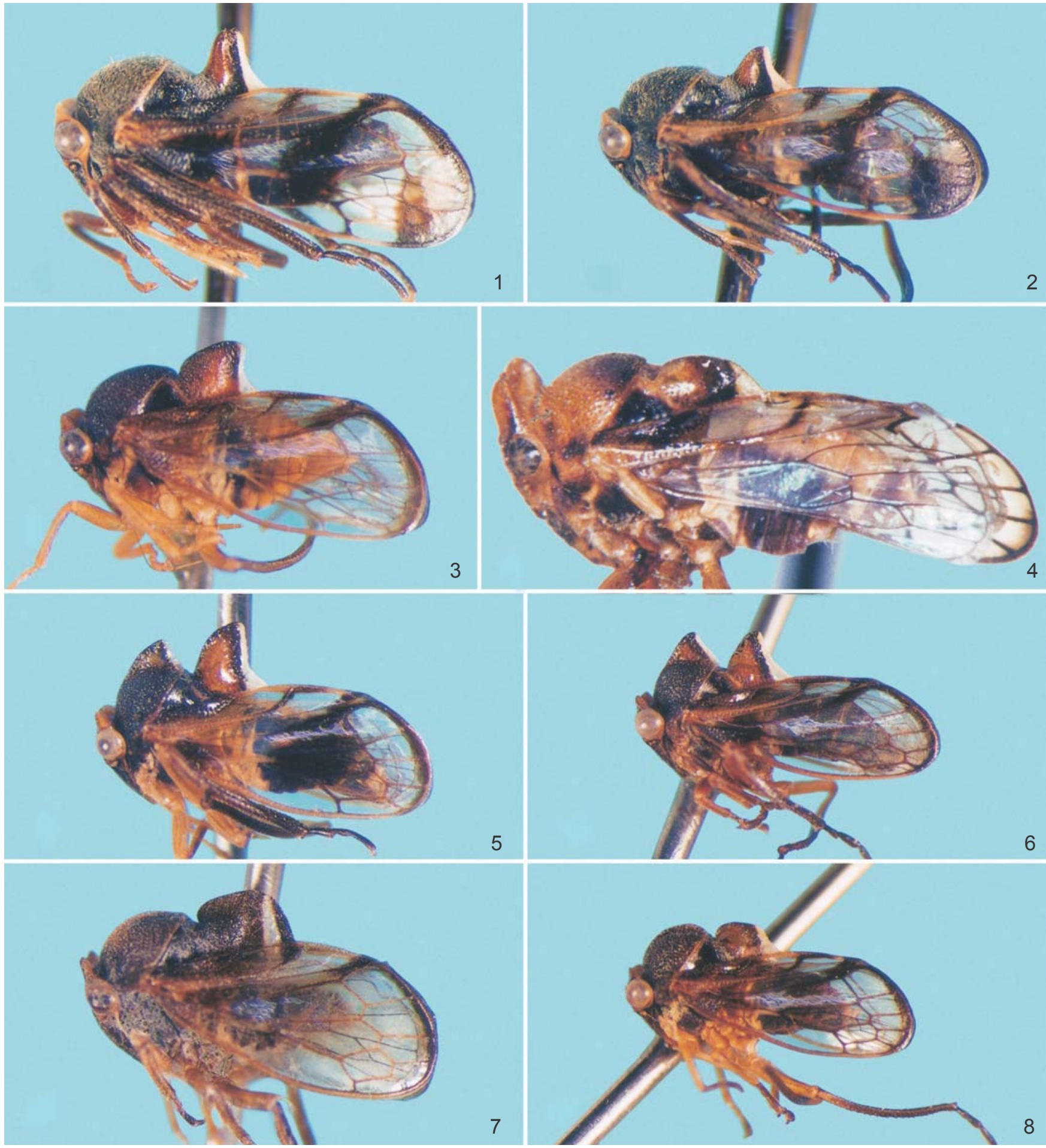

Figs. 1-8. Espécies de Lophyraspis Stål: 1, L. muscaria (Fabricius, 1803) fêmea; 2, macho. 3, L. pygmaea (Fabricius, 1803) fêmea. 4, Mina spinosa Funkhouser, 1930 (holótipo fêmea, USNM). 5, L. scutellata (Fabricius, 1803) fêmea; 6, macho. 7, L. fenestrata sp. nov. (holótipo fêmea). 8, L. diminuta sp. nov. (holótipo macho). 


\section{Lophyraspis spinosa (Funkhouser, 1930) comb. nov.} (Fig. 4)

Mina spinosa Funkhouser, 1930: 409 (localidade-tipo: Equador); Metcalf \& Wade, 1965: 1541; McKamey, 1998: 18 (cat.).

Diagnose. Cabeça com a parte superior do vértice fortemente elevada, sobrepassando a altura do pronoto, distintamente bilobada. Escutelo pouco mais baixo que o pronoto, não formando crista, a parte posterior laminar e translúcida. Tégminas vítreas, com um leve enfumaçamento no bordo distal.

Material examinado. Apenas uma fotografia (slide) do holótipo (USNM)

FunkHouser (1930) descreveu Mina spinosa com base em um exemplar fêmea do Equador. Na descrição salientou que o escutelo apresentava um prolongamento posterior em forma de um delgado espinho (daí o nome específico), caráter esse representado na sua figura 6. Examinando a fotografia do holótipo, observamos que o escutelo não apresenta tal prolongamento ("hairlike"). O autor deve ter se equivocado, interpretando a margem anal das tégminas, que é levemente engrossada e bem pigmentada, como sendo um prolongamento do ápice do escutelo. Já, na figura 126 apresentada por FunKHOUSER (1951), o prolongamento não está representado. A fotografia do holótipo não permite uma visualização perfeita para se afirmar se o pronoto tem ou não a carena média dorsal pronunciada. Tentativamente consideramos como não tendo tal caráter.

B. Pronoto com carena média pronunciada ou em forma de crista.

\section{Lophyraspis scutellata (Fabricius, 1803)}

(Figs. 5, 6)

Tettigonia scutellata Fabricius, 1803: 44 (localidade-tipo: América do Sul).

Lophyraspis scutellata; Funkhouser, 1927: 432. Metcalf \& Wade, 1965:1538; McKamey, 1998: 17 (cat.).

Lophyraspis cristata Stål, 1869: 56 (localidade-tipo: América do Sul). Syn nov.

Biturritia cristata; Metcalf \& Wade, 1965: 1530; McKamey, 1998: 16 (cat.).

Lophyraspis armata Haviland, 1925: 262 (localidade-tipo: Guiana); Funkhouser, 1927: 432. Syn. nov.

Gerridius armatus; Metcalf \& Wade, 1965: 1539; McKamey, 1998: 17 (cat.).

Diagnose. Carena média do pronoto elevada em forma de uma pequena crista, mais ou menos triangular, com a parte posterior translúcida. Escutelo elevado pouco acima da altura da carena pronotal, em uma crista triangular, aproximadamente tão larga quanto alta, margem posterior laminar, translúcida e incolor. Tégminas vítreas, com áreas escuras na base, no disco, e margeando a parte distal.

Material examinado. BRASIL. Pará: Belém. Maranhão: Santa
Luzia. Mato Grosso: Sinop. (DZUP).

STÅL (1869), quando redescreveu L. scutellata Fabricius, comentou que entre os exemplares desta espécie havia um que se destacava pelo tamanho das cristas, tanto do pronoto como do escutelo, ao qual dava o nome de L. cristata. $\mathrm{Na}$ descrição diz que a crista do escutelo é tão larga quanto alta. Verificamos que há dimorfismo sexual em L. scutellata, as fêmeas são maiores e as cristas do pronoto e do escutelo também são mais desenvolvidos. McKameY (1998) preferiu incluí-la em Biturritia, o que discordamos.

Haviland (1925) descreveu uma espécie da Guiana que chamou de L. armata, provavelmente devido às cristas dorsais; comentou que era muito semelhante à L. cristata. Consideramos, também, como um novo sinônimo.

\section{Lophyraspis fenestrata sp. nov.}

(Fig. 7)

Medidas (em mm). Holótipo fêmea. Comprimento total, 4,40; distância do metopídio ao ápice do escutelo, 2,40; altura máxima do escutelo, 0,80; largura da cabeça, 1,48; largura entre os úmeros, 1,80 .

Coloração geral castanho-escura, levemente mais clara no vértice, ao longo da linha média do pronoto e pernas, exceto as tíbias posteriores; escutelo com uma faixa vertical incolor, translúcida, mais ou menos triangular, pouco antes do ápice; tégminas levemente enfumaçadas, com o contorno apical mais escuro.

Cabeça mais ou menos triangular. Vértice amplo, levemente deprimido entre os ocelos, com a margem superior elevada acima dos olhos, laminar e largamente bilobada; olhos semiglobosos, pouco salientes; ocelos inconspícuos, localizados logo abaixo da linha imaginária que tangencia a parte superior dos olhos, mais próximos destes que da linha mediana; lóbulos suprantenais pequenos, com os bordos arredondados. Pósclípeo e anteclípeo levemente intumescidos, pouco diferenciados. Rostro longo, com o artículo distal, na sua totalidade, ultrapassando as coxas posteriores.

Pronoto convexo, com a carena média dorsal, embora baixa, bem vincada. Escutelo, em vista lateral, elevado em uma crista mais ou menos quadrangular, intumescida na base e de altura pouco acima do nível do pronoto, posteriormente em forma de lâmina delgada.

Tégminas vítreas, com veias delgadas, porém, bem visíveis; uma célula discoidal e cinco apicais; transversas s, s-m e m-cu presentes. Asas posteriores hialinas, com quatro células apicais.

Macho. Desconhecido.

Holótipo fêmea. "Manaus, AM [BRASIL]/ Caracará, Km 14/ 10X-87/ N. Silva leg." (DZUP). Parátipos: 2 fêmeas, com os mesmos dados do holótipo (DZUP).

Comentários. Esta espécie é intermediária entre L. pygmaea 
e L. scutellata; difere da primeira pela presença da carena média dorsal no pronoto e, da segunda, por apresentar tanto a carena média dorsal como o escutelo pouco elevados, sem formar cristas evidentes. As características que definem a espécies são: pronoto com carena média dorsal bem evidente, porém sem formar uma crista; escutelo pouco elevado, mais ou menos quadrangular, provido de uma estreita faixa vertical incolor, translúcida, pré-apical. Característica esta que sugeriu o seu nome.

\section{Lophyraspis diminuta sp. nov.} (Fig. 8)

Medidas (em mm). Holótipo macho. Comprimento total, 3,48; distância do metopídio ao ápice do escutelo, 1,70; altura máxima do escutelo, 0,44; largura da cabeça, 1,26; largura entre os úmeros, 1,32 .

Coloração geral castanho-escura, levemente mais clara no escutelo; este com a margem posterior translúcida, incolor; pernas amareladas, exceto os tarsos e tíbias posteriores, castanho-escuros; tégminas escurecidas na base e no contorno apical, incluindo o ápice do clavo e margem anal; abdome escuro, com uma estreita faixa transversa esbranquiçada na base.

Cabeça mais ou menos triangular. Vértice amplo, levemente deprimido entre os ocelos, margem superior elevada acima dos olhos, largamente sinuosa, porém, sem formar lobos distintos; olhos semi-globulares, pouco salientes; ocelos bem visíveis, localizados logo abaixo da linha imaginária que passa tangente à parte superior dos olhos, mais próximos destes que da linha mediana; lóbulos suprantenais obsoletos, com os bordos arredondados. Pós-clípeo e anteclípeo muito pouco intumescido, a sutura transclipeal pouco evidente. Rostro ultrapassando as coxas posteriores em menos da metade do artículo distal.

Pronoto convexo, com a carena média dorsal baixa, porém, bem vincada. Escutelo elevado, em vista lateral, subquadrangular, na mesma altura do pronoto, com a margem posterior laminar.

Tégminas vítreas, com venação distinta como nas demais espécies.

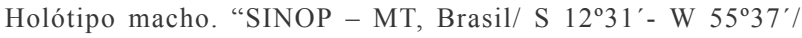
X.1974 - M. Alvarenga” (DZUP).
Comentários. Esta espécie é muito parecida com L. spinosa mas difere pelos seguintes caracteres: a cabeça com o bordo superior do vértice muito pouco projetado acima dos olhos, não formando lobos distintos; o pronoto apresenta a carena média bem evidente; o escutelo é um pouco mais elevado, porém, não passando do nível do pronoto.

\section{REFERÊNCIAS}

Baker, C. F. 1907. Notes and descriptions of Membracidae. The Canadian Entomologist 39 (4): 114-118.

Deitz, L. L. \& C. H. Dietrich. 1993. Superfamily Membracoidea (Homoptera: Auchenorrhyncha). I. Introduction and revised classification with new family-group taxa. Systematic Entomology 18: 287-296.

Fabricius, J. C. 1803. Rhyngota. Systema Rhyngotorum secundum ordines, genera, species adiectis synonymis, locis, observationibus, descriptionibus: 1-314.

Fowler, W. W. 1896. Order Rhynchota. Suborder Hemiptera-Homoptera. (Cont.). Biologia Centrali-Americana 2: 161-168.

Funkhouser, W. D. 1930. New genera and species of Neotropical Membracidae. Journal of the New York Entomological Society 38: 405-421.

Funkhouser W. D. 1951. Homoptera. Fam. Membracidae. In: P. Wytsman (Ed.). Genera Insectorum, $\mathbf{2 0 8}^{\mathrm{e}}$ fasc. Bruxelles, Louis Desmet Verteneuil: 1- 383 .

Goding, F. W. 1927. Revision of the Membracidae of South America and Antilles. Journal of the New Yortk Entomological Society 35: $183-191$.

Haviland, M. D. 1925. The Membracidae of Kartabo Bartica District, British Guiana. With descriptions of new species and bionomical notes. Zoologica 6 (3): 229-290.

Mckamey, S. H. 1997. Nomenclatural changes in the Membracidae and Aetalionidae (Hemiptera: Membracoidea): species-group names and Sphongophorus Fairmaire, revised status. Steenstrupia 22: 111 .

Mckamey, S. H. 1998. Taxonomic catalogue of the Membracoidea (exclusive of leafhoppers): second supplement to fascicle I Membracidae of the General Catalogue of the Hemiptera. Memoirs of the American Entomological Institute 60: 1-377.

Olivier, G. A. 1790. Cigale. Cicada. Encyclopédie méthodique d'histoire naturelle des insectes 4: 1-331.

STÅ, C. 1869. Hemiptera Fabriciana. Öfversigt af Kongl. Vetenskaps-Akademiens Förhandlingar 8: 18-58.

Stoll, C. 1788. Cigales. Représentation exactement colorée d'après nature des cigales, qui se trouvent dans les quatre parties du monde, l'Europe, l'Asie, l'Afrique, et l'Amérique; rassemblées et décrites 1788: 1-124. 